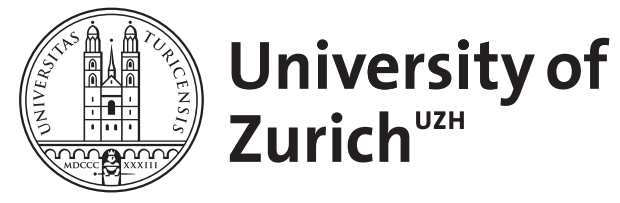

Zurich Open Repository and Archive

University of Zurich

University Library

Strickhofstrasse 39

CH-8057 Zurich

www.zora.uzh.ch

Year: 2012

\title{
Akute Fremdkörperverlegung der Atemwege bei Kindern
}

Weiss, M ; Tomaske, M

DOI: https://doi.org/10.1007/s10049-011-1513-1

Posted at the Zurich Open Repository and Archive, University of Zurich

ZORA URL: https://doi.org/10.5167/uzh-67345

Journal Article

Published Version

Originally published at:

Weiss, M; Tomaske, M (2012). Akute Fremdkörperverlegung der Atemwege bei Kindern. Notfall + Rettungsmedizin, 15(2):111-116.

DOI: https://doi.org/10.1007/s10049-011-1513-1 


\section{Leitthema}

Notfall Rettungsmed 2012 · 15:111-116

DOI 10.1007/s10049-011

Online publiziert: 1. Februar 2012

๑) Springer-Verlag 2012

\section{Redaktion}

M. Weiss, Zürich

T. Nicolai, München

M. Weiss ${ }^{1}$ M. Tomaske ${ }^{2}$

${ }^{1}$ Anästhesieabteilung, Universitäts-Kinderkliniken, Zürich

${ }^{2}$ Klinik für Kinder und Jugendliche, Stadtspital Triemli Zürich

\section{Akute Fremdkörperverlegung der Atemwege bei Kindern}

fallkonsultationen bei Kindern in den USA dar [2].

Frühzeitige Diagnose und unverzügliche Entfernung des Fremdkörpers sind Voraussetzung, um das Kind vor schwerwiegender Morbidität oder Mortalität $\mathrm{zu}$ bewahren. Entsprechend ist ein fundiertes Wissen hinsichtlich Diagnostik, Überwachung und Management der akuten Fremdkörperaspiration für alle in der Kindernotfallmedizin involvierten Personen von entscheidender Bedeutung.

\section{Diagnostik}

\section{Anamnese}

mit einem Fremdkörper stellt eine akute lebensbedrohliche Situation dar. Sie tritt am häufigsten bei Kleinkindern in den ersten 3 Lebensjahren auf. Knaben sind doppelt so häufig betroffen wie Mädchen. Meistens handelt es sich bei den aspirierten Fremdkörpern um Esswaren (80\%) mit einer Prädominanz von Nüssen (50\%). Die Aspiration in die rechte Lunge ist fast doppelt so häufig wie in die linke [1]. Akut lebensbedrohliche Situationen mit Erstickungsgefahr ergeben sich bei laryngealer oder trachealer Lage des Fremdkörpers.

\section{》) Frühzeitige Diagnose}

und schnelle Entfernung des

\section{Fremdkörpers sind entscheidend}

Das Risiko für eine tödliche Fremdkörperaspiration für Kinder unter 4 Jahren liegt bei 0,7/100.000 Einwohner/Jahr. Das Aspirationsereignis stellt ein nicht seltenes notfallmedizinisches pädiatrisches Krankheitsbild mit jährlich >17.000 Not-
Im Allgemeinen handelt es sich um Säuglinge und Kleinkinder, welche aus Neugier kleine Gegenstände in den Mund nehmen, Nüsse un- oder ungenügend zerkaut im Mund haben und beim Spielen oder Erschrecken in die oberen und unteren Atemwege aspirieren. Bei größeren Kindern sind es Gegenstände, welche zuvor zwischen den Lippen festgehalten oder auf der Zunge balanciert wurden (Schrauben, Nadeln etc.).

Typisch beim akuten Aspirationsereignis ist ein plötzlicher starker Husten (Hustenanfall) und/oder Würgen. Gegebenfalls gehen diese Symptome mit einer Zyanose und/oder Atemnot einher.

Persisiert der Fremdkörper in den Atemwegen, resultiert ein beträchtlicher Hustenreiz, begleitet von einem inspiratorischen Stridor (obere Atemwege) oder einem exspiratorischem Giemen (untere Atemwege). Kleinere Fremdkörper können in kleinere Bronchien gesogen werden und zunächst unbemerkt bleiben.
Dies kann im Akutstadium zum Ventilmechanismus mit poststenotischer Überblähung führen sowie sekundär als chronischer Husten bei poststenotischer Pneumonie imponieren.

\section{Befunde}

Typische Befunde sind neben dem akuten Hustenanfall Atemnot, ein in- und/oder exspiratorischer, vom Ohr hörbarer Stridor sowie ein unilateral abgeschwächtes Atemgeräusch mit verminderter Thoraxexkursion begleitet von Giemen.

Einen weiteren Hinweis für einen Fremdkörper kann das Thorax-Röntgenbild mit Überblähung der betroffenen Seite und Mediastinalshift zur nichtbetroffenen Seite geben. Die Aufnahme erfolgt bei kooperativem Kind möglichst in einer Exspiratonsstellung. Die Sensitivität und Spezifität des Thorax-Röntgenbilds, einen Fremdkörper zu diagnostizieren bzw. auszuschließen, liegt bei je ca. 60-70\% [3]. Organische Fremdkörper sind nicht röntgendicht und nur selten ist im ThoraxRöntgenbild der Fremdkörper zu sehen.

Während die klinische Trias mit akutem Würgen/Husten, Giemen und einseitig abgeschwächten Atemgeräuschen eine hohe Spezifität (96\%) für einen Atemwegsfremdkörper aufweist, kann jedoch nach einer akuten Hustenattacke ohne sonstige pathologische klinische Befunde nicht ausgeschlossen werden, dass sich (noch) ein Fremdkörper in den Atemwegen befinde. Da respiratorische Infekte im Kindesalter sehr häufig sind, kann ein Hustenanfall mit starkem Giemen als Asthmaanfall oder obstruktive Bronchitis fehlgedeutet werden $[1,4]$. 
Da bei einem akuten Hustenanfall weder einzelne noch Kombinationen von anamnestischen oder klinischen Variablen oder das Thorax-Röntgenbild einen Fremdkörper in den Atemwegen ausschließen können, bedarf es immer einer bronchoskopischen Kontrolle [1]. Die Bronchoskopie in Allgemeinanästhesie hat in geübten Händen keine relevante Morbidität, während die Morbidiät und Mortalität eines verpassten Atemwegsfremdkörpers (sekundäre Dislokation mit akuter Atemwegsverlegung, sekundäre poststenotische Pneumonie, Abszedierung, Sepsis und konsekutive erschwerte bronchoskopische Entfernung) beträchtlich sind [5].

\section{Differenzialdiagnose}

Die kindlichen Atemwege sind aufgrund ihrer Anatomie und dem geringeren Durchmesser anfällig für eine Beeinträchtigung der Atemwege und einem potenziell lebensbedrohlichen Krankheitsbild. Insbesondere bei fehlender Anamnese eines Aspirationsereignisses bzw. Hustenanfalls müssen an weitere Differentialdiagnosen in Betracht gezogen werden [6, 7]: Bei akutem Giemen als Leitsymptom ist eine akute Obstruktion der Bronchien und Bronchiolen durch andere, altersabhängige Ätiologien möglich, wie z.B. die obstruktive Bronchitis im Säuglings und Kleinkindesalter sowie das infektassoziierte oder allergische Asthma im Kleinkind- und Schulkindalter.

Bei akutem inspiratorischem Stridor als Leitsymptom ist differenzialdiagnostisch an eine Laryngotracheitis (Pseudo-Krupp), eine Epiglottitis, eine akut-bakterielle Tracheitis zu denken. Bei in- und exspiratorischem Stridor ist eine Raumforderung im Pharynx/Larynx oder gar ein großer ösophagealer Fremdkörper möglich.

Die definitive Diagnose erfordert im Zweifelsfalle wiederum eine Endoskopie der Atemwege.

\section{Transport und Überwachung}

Nicht selten beruhigt sich das Zustandsbild des Patienten nach einem akuten Erstickungsanfall (Husten; Würgen) von selbst oder die Symptome sind gänzlich verschwunden, bis das Rettungsteam eintrifft oder die Eltern mit dem Kind einen Arzt aufgesucht haben. Patienten im Kindesalter mit einem akuten Erstickungsereignis/Hustenanfall müssen auf einer Notfallstation untersucht werden, welche sich mit pädiatrischen Krankheitsbildern und deren Behandlung auskennt, auch wenn die Symptome gänzlich verschwunden sind.

Bei Persistenz von Symptomen (Husten, Stridor oder Atemnot) muss der Transport in die Klinik von einem Notarzt begleitet werden, da jederzeit durch Hustenstöße der Fremdkörper dislozieren und zu einer vital bedrohlichen Verlegung der Glottis oder Trachea führen kann. Insbesondere Kinder mit in- und expiratorischem Stridor und/oder Atemnot sind akut gefährdet.

\section{》) Bei Persistenz von Symptomen muss beim Transport in die Klinik ein Notarzt anwesend sein}

Symptomatische Kinder werden mit der Ambulanz in eine Zentrumsklinik mit Kinderbronchoskopiedienst transportiert und ohne weitere Bilddiagnostik in den Endoskopie-/Operationsraum gebracht, möglichst in den beruhigenden Armen der Mutter/des Vaters und in einer aufrechten Position sitzend. Eine entsprechende Voranmeldung in der avisierten Klinik versteht sich von selbst. Ebenso soll die Anlage eines venösen Zugangs bei diesen Kindern erst in Endoskopiebereitschaft erfolgen, um unnötige Aufregung mit der Gefahr einer Fremdkörperdislokation zu vermeiden und keine Zeit zu verlieren. Im Bedarfsfall kann mit einer intraossären Leitung genügend schnell und zuverlässig ein intravasaler Zugang etabliert werden [8]. Die Patienten sollen mit pulsoximetrischer Überwachung und vorgehaltenem Sauerstoff unter ärztlicher Begleitung in die Klinik transportiert werden.

Management der akuten Fremdkörperverlegung der Atemwege

\section{Szenario I: waches Kind, anamnestisch Aspirationsereignis, ohne Symptome}

Kinder mit anamnestisch akutem Hustenanfall, Würgen oder Erstickungser- eignis werden auch bei Fehlen von Symptomen und Befunden in eine pädiatrische Notfallstation überwiesen und bronchoskopisch auf Vorliegen eines „stillen“ Fremdkörpers in den unteren Atemwegen kontrolliert. In den meisten Fällen erfolgt die Bronchoskopie ambulant.

Jedes Kind muss nach einem akuten schweren Hustenanfall - auch wenn die Symptome verschwunden sind - einer Bronchoskopie unterzogen werden, um verbleibende Fremdkörper zu entfernen und damit eine sekundäre Dislokation in die oberen Atemwege bzw. poststenotische Komplikationen zu verhindern

Szenario II: waches Kind mit akuter Fremdkörperverlegung der Atemwege, Husten mit oder ohne Atemnot

Kinder mit einem Aspirationsereignis, persistierendem Husten und klinischen Befunden werden mit ärztlicher Begleitung in die Klinik eingewiesen und baldmöglichst, $d$. $h$. nach Abwarten der vorgegebenen Nüchternzeit, einer Bronchoskopie unterzogen. Kinder mit Dyspnoe sowie mit Verdacht auf laryngeale/tracheale Fremdkörper werden ungeachtet der Nüchternzeit schnellstmöglichst endoskopiert. Falls dies nicht möglich ist, müssen sie auf einer Intensivstation überwacht werden.

\section{Szenario III: waches Kind mit akuter Fremdkörperverlegung der Atemwege, Erstickungsgefahr und effektivem Husten}

Beim Kind, das bei Bewusstsein ist (wach, ansprechbar, Weinen oder verbale Reaktion auf Ansprache) und effizient hustet und damit auch atmet, sollen vor Ort keine Extraktionsmanöver durchgeführt werden. Dies gilt deshalb, um eine Dislokation des Fremdkörpers in eine noch ungünstigere Lage innerhalb der Atemwege zu vermeiden. Bei drohender Erstickungsgefahr muss das Kind angehalten werden, weiterhin kräftig zu husten. Es wird unverzüglich mit Notarzt in Intubationsbereitschaft und mit Voranmeldung in die nächste Zentrumsklinik mit einem Kinderbronchoskopiedienst gefahren. 


\section{Weiss $\cdot$ M. Tomaske \\ Akute Fremdkörperverlegung der Atemwege bei Kindern}

\section{Zusammenfassung}

Hintergrund. Die Fremdkörperverlegung

der Atemwege beim Kind stellt eine akut lebensbedrohliche respiratorische Notfallsituation dar. Es sind v. a. Kleinkinder in den ersten 3 Lebensjahren betroffen. Esswaren, insbesondere Nüsse, sind die am häufigsten aspirierten Fremdkörper. Akute Erstickungsgefahr besteht bei laryngealer und trachealer Lage des Fremdkörpers.

Klinik und Differenzialdiagnose. Typisch für eine Fremdkörperaspiration ist der plötzlich einsetzende Husten und/oder Würgen, ggf. begleitet von Atemnot und Stridor. Persistiert der Fremdkörper, resultiert ein beträchtlicher Husten. Laryngeal und tracheal gelegene Fremdkörper können zur respiratorischen Insuffizienz, Bewusstlosigkeit und Kreislaufstillstand führen. Differenzialdiagnostisch muss an eine schwere bronchiale, infektiöse oder allergische Obstruktion der oberen Atemwege oder an einen großen Fremdkörper im Ösophagus gedacht werden.

Erstversorgung. Kinder mit akuter Fremdkörperverlegung der Atemwege mit oder ohne Dyspnoe/Erstickungsgefahr, jedoch noch mit effektivem Husten und Atmung, sollen ohne Extraktionsmanöver vor Ort baldmöglichst der Bronchoskopie zugeführt werden. Bei wachen Kindern mit ineffektivem Hustenstoß muss mit Rückenschlägen, Thoraxkompression beim Säugling bzw. abdomineller Kompression beim > 1 Jahr alten Kind versucht werden, den Fremdkörper in eine günstigere Lage zu dislozieren. Das bewusstlose, ateminsuffiziente Kind wird beatmet. Erfolgt damit keine Reaktion, wird mit der Herzmassage bzw. der kardiopulmonalen Reanimation begonnen. Der erfahrene Ersthelfer sollte mittels Laryngoskopie einen laryngealen Fremdkörper entfernen bzw. mit einem Endotracheltubus einen trachealen Fremdkörper tiefer in einen Hauptstammbronchus schieben und das Kind unilateral beatmet der Notfallendoskopie zuführen. Jedes Kind muss nach einem akuten schweren Hustenanfallauch wenn die Symptome verschwunden sind - einer Bronchoskopie unterzogen werden, um potenzielle Fremdkörper zu entfernen und damit eine sekundäre Dislokation in die oberen Atemwege bzw. poststenotische Komplikationen zu verhindern.

\section{Schlüsselwörter}

Fremdkörperaspiration · Kinder .

Respiratorische Insuffizienz .

Atemwegsobstruktion · Bronchoskopie

\section{Acute foreign body obstruction of the airway in children}

\section{Abstract}

Background. Acute airway obstruction by a foreign body in children is a life-threatening emergency respiratory condition. Respiratory foreign body aspiration mostly occurs in children up to 3 years of age. Food and in particular nuts are the most commonly ingested respiratory foreign bodies. Acute airway obstruction is present with a laryngeal or tracheal position of the foreign body.

Diagnosis. Acute, sudden and severe coughing and/or choking sometimes accompanied by stridor and dyspnea are characteristic of a respiratory foreign body aspiration. Foreign bodies with a laryngeal or tracheal position may rapidly lead to respiratory insufficien$c y$, decreased levels of consciousness and circulatory arrest. Severe bronchial obstruction, infectious or allergic upper airway obstruc- tion or airway obstruction caused by a large esophageal foreign body should be excluded. Management. Children with acute airway obstruction by a foreign body with or without dyspnea and danger of suffocation but still with effective coughing and breathing have to be transferred by an emergency physician to a pediatric bronchoscopy unit without undertaking any external maneuvers at the scene. In awake children with inefficient coughing back slapping and thoracic compression in infants or abdominal compression in children aged less than 1 year old have to be performed to help the child to expel or to move the obstructing object to a more advantageous position. In children with asphyxia and loss of consciousness artificial ventilation should be started and if there is no re- action with ventilation, extrathoracic cardiac compression or cardiopulmonary resuscitation must be started. Experienced first aiders should inspect the larynx by direct laryngoscopy to remove or push a foreign body down into a main stem bronchus and ventilate the other lung using an endotracheal tube. Every child suffering from acute severe coughing or choking, even without symptoms must undergo bronchoscopic control to exclude or remove a residual foreign body, which can potentially cause secondary airway obstruction or poststenotic complications.

Keywords Foreign body aspiration - Children . Respiratory compromise - Airway obstruction · Bronchoscopy

\section{Szenario IV: waches Kind mit akuter Fremdkörperverlegung der Atemwege, Erstickungsgefahr und ineffektivem/fehlendem Husten}

Beim Kind mit Fremdkörperverlegung und Erstickungsgefahr, das noch bei Bewusstsein ist, aber nur ineffektiv oder nicht mehr hustet (stilles oder leises Husten, kann nicht sprechen, kann nicht richtig atmen, Zyanose) werden
Rückenschläge verabreicht. Der Helfer hält in kniender oder sitzender Position den Säugling in Bauchlage mit dem Kopf nach unten, damit die Entfernung des Fremdkörpers durch die Schwerkraft unterstützt wird. Dabei dürfen die Halsweichteile nicht komprimiert werden, da sie eine Atemwegsobstruktion verschlimmern. Es werden mit dem Handballen bis zu 5 scharfe Schläge auf die Mitte des Rückens zwischen die Schulterblätter. verabreicht. Das Ziel besteht darin, die
Verlegung mit jedem einzelnen Schlag zu beseitigen, und nicht, alle 5 Schläge zu verabreichen ([9]; • Abb. 1). Bei Kindern $>1$ Jahr sind die Rückenschläge effektiver, wenn das Kind in eine Kopftieflage gebracht wird. Ein kleines Kind kann wie ein Säugling auf dem Schoß des Helfers gelagert werden. Falls dies nicht möglich ist, sollte das Kind in eine vornüber gebeugte Position gebracht und die Rückenschläge sollten von hinten verabreicht werden ([9]; • Abb.2). Kann 

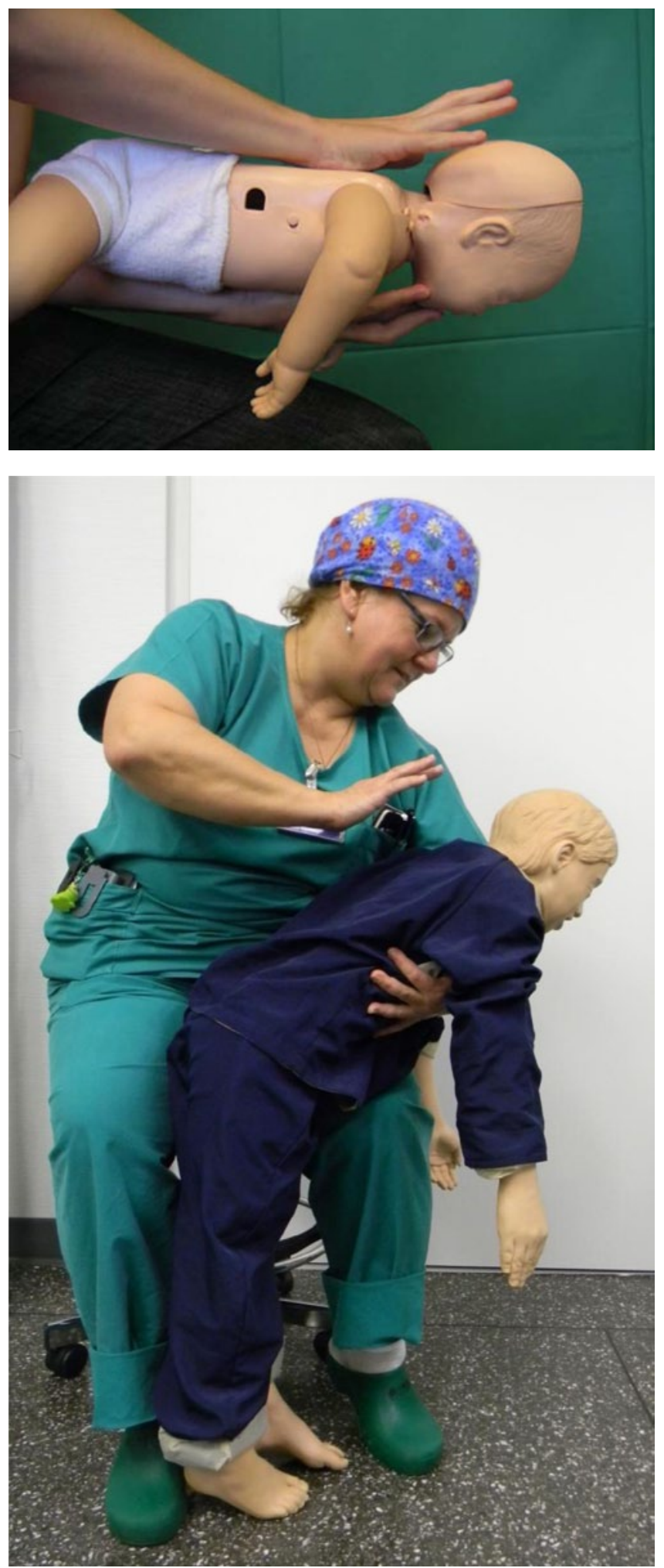

durch die Rückenschläge die Fremdkörperverlegung nicht beseitigt werden und das Kind ist weiterhin bei Bewusstsein, so werden beim Säugling 5 Thoraxkompressionen in Kopftieflage durchgeführt ([9]; • Abb. 3). Bei Kindern über 1 Jahr
Abb. $2<$ Rückenschläge beim größeren Kind mit akuter Fremdkörperverlegung der Atemwege und ineffizientem Husten/Atmung

Abb. $1<$ Rückenschläge beim wachen Säugling mit akuter Fremdkörperverlegung der Atemwege und ineffizientem Husten/Atmung

werden bis zu 5 abdominelle Kompressionen (Heimlich-Manöver, • Abb.4) angewandt. Der Helfer steht oder kniet hinter dem Kind und legt seine Arme unter die des Kindes und umfasst seinen Rumpf. Die eine Hand des Helfers wird als Faust zusammengeballt zwischen Nabel und Xiphoid auf dem Bauch platziert. Die Hand wird mit der anderen gefasst und scharf nach innen und oben gezogen. Das Manöver wird bis zu 5-mal wiederholt. Der ruckartige Druck darf nicht auf das Xiphoid oder den unteren Brustkorb ausgeübt werden [9]. Die erzeugten künstlichen Hustenstöße sollen helfen, den Fremdkörper auszustoßen oder in eine günstigere Lage zu bringen. Beim Säugling dürfen keine abdominellen Kompressionen (Heimlich-Manöver) durchgeführt werden (Rupturgefahr der noch großen Leber und Milz).

Nach Abschluss der Manöver wird das Kind neu evaluiert. Falls sich die Situation nicht verbessert hat und das Kind noch bei Bewusstsein ist, werden die Abfolgen von „Rückenschlägen und Thoraxkompressionen" wiederholt und es wird Hilfe angefordert. Das Kind darf nicht alleine gelassen werden. Kann der Fremdkörper auf diese Art ausgestoßen oder günstig disloziert werden, muss das Kind in Begleitung eines Notarztes in die Klinik transportiert und dort bronchoskopisch auf verbleibende oder einen stillen Fremdkörper hin untersucht werden.

\section{Szenario V: bewusstloses Kind mit akuter Fremdkörper- verlegung der Atemwege}

Wird oder ist das Kind mit akuter Fremdkörperverlegung der Atemwege bewusstlos, wird es mit dem Rücken auf eine feste, flache Unterlage gelegt und Hilfe herbeigerufen. Das Kind darf unter keinen Umständen alleine gelassen werden.

1. Freimachen der Atemwege: Der Mund wird geöffnet und nach einem fassbaren Fremdkörper inspiziert und, falls vorhanden, einmalig mit Auswischen entfernt. Es werden keine blinden oder wiederholten Auswischversuche durchgeführt (Zeitfaktor und Gefahr der Dislokation des Fremdkörpers tiefer in den Atemweg, Verletzungen).

2. Beatmung: Das Kind wird nach Halsüberstrecken und Kinn anheben 5-mal beatmet (Mund oder Maskenbeatmung). Falls keine Beatmung möglich ist, wird der Kopf vor dem nächstem Versuch nochmals optimiert positioniert. Kann das Kind beatmet werden, 

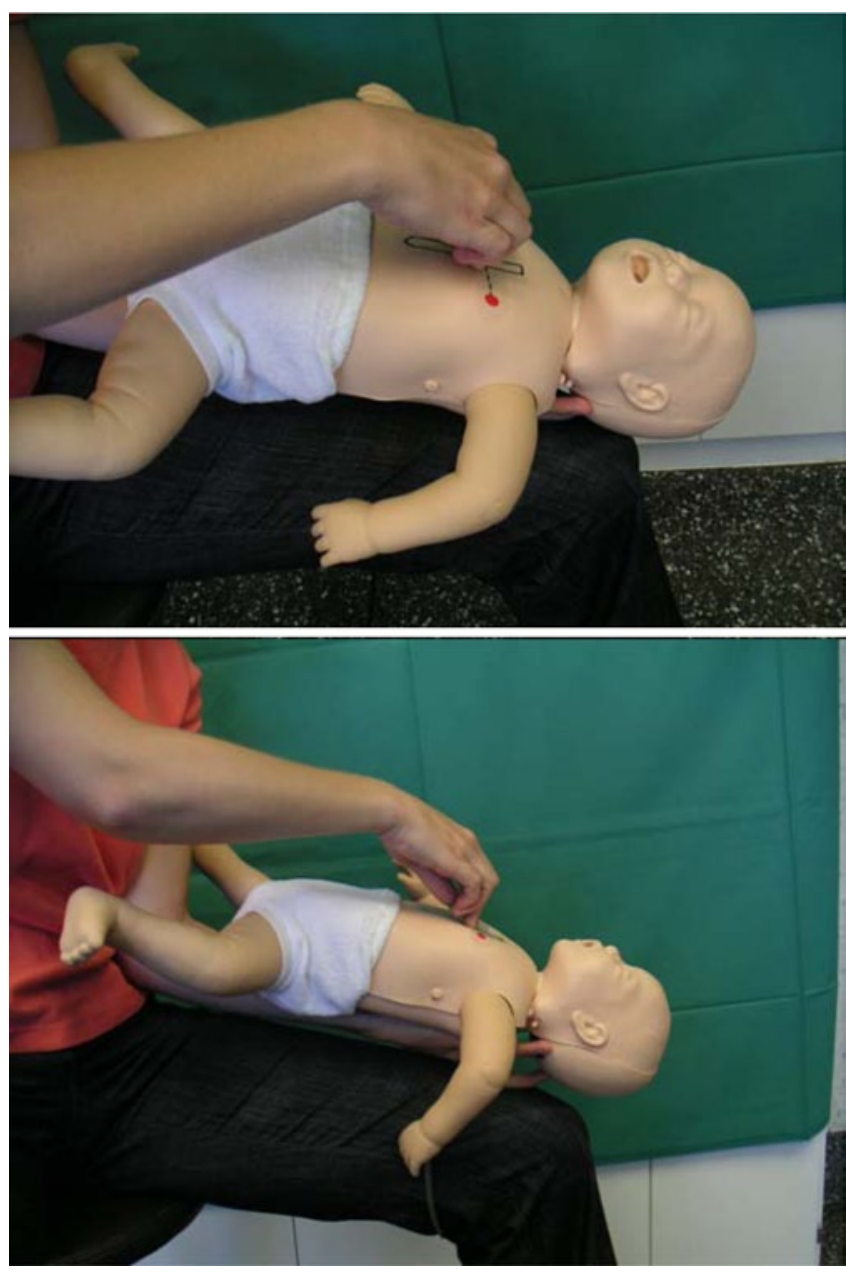

Abb. $3<$ Thoraxkompression beim wachen Säugling mit akuter Fremdkörperverlegung der Atemwege und ineffizientem Husten/Atmung

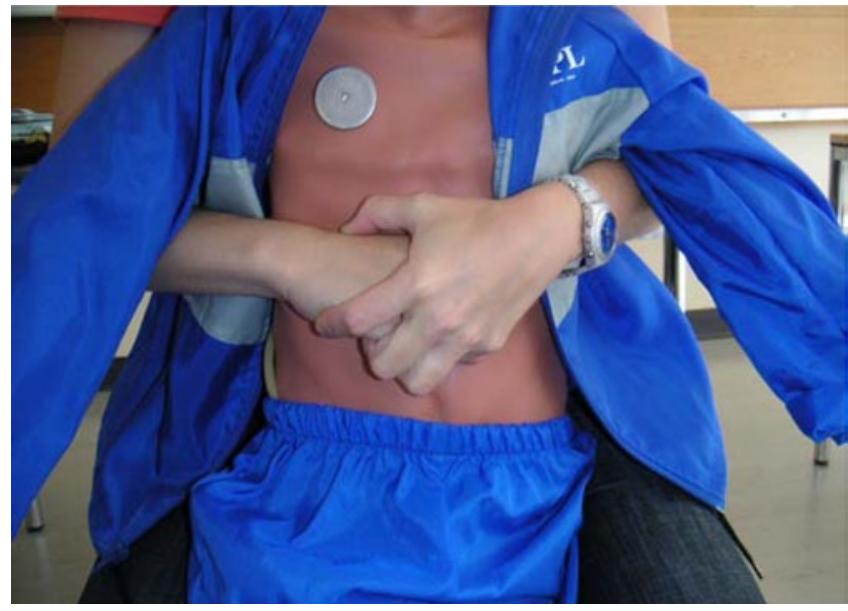

Abb. $4<$ Abdominelle Kompression beim wachen Kind (> 1 Jahr) mit akuter Fremdkörperverlegung der Atemwege und ineffizientem Husten/Atmung

wird abgewartet, ob Bewegung, Husten oder gar Spontanatmung wieder eintritt. Es kann unter Maskenbeatmung oder unterstützender Beatmung in die Klinik gebracht werden bzw. der Endoskopie zugeführt werden.

3. Herzdruckmassage und kardiopulmonale Reanimation: Zeigt das Kind nach initialen Beatmungsversuchen kei- druckmassage hilft, den Fremdkörper in eine günstigere Position zu verschieben und einen minimalen Kreislauf aufrechtzuerhalten. Sobald die Atemwegsverlegung behoben wurde, das Kind suffizient atmet und das Bewusstsein wiedererlangt, wird es in Seitenlage gebracht [9].

4. Spezielle Techniken für den geübten Notarzt oder in der Klinikaufnahme:

a) Das Kind kann trotz Repositionierung des Kopfes und 1-Hand-2Personen-Masken-Beatmung nicht suffizient beatmet werden: Je nach Erfahrung und Ausrüstung kann eine direkte Laryngoskopie erfolgen, um ggf. einen pharyngeal/laryngeal gelegenen Fremdkörper mit der Magil-Zange unter Sicht zu entfernen [8]. Supraglottische Atemwegshilfen sind kontraindiziert, da diese - blind eingeführt den Fremdkörper tiefer schieben können.

b) Das Kind nach akuter Fremdkörperaspiration kann mit der Maske suffizient beatmet werden: Auf eine Intubation oder weitere Manöver sollte verzichtet werden. Unter Maskenbeatmung wird es in die Klinik transportiert und dort unverzüglich der Endoskopie zugeführt.

c) Es ist kein Fremdkörper im Rachen-Kehlkopf-Bereich des Kindes zu sehen und es ist anzunehmen, dass der Fremdköper subglottisch oder tracheal liegt: Je nach Erfahrung und Ausrüstung kann ein Versuch erfolgen, mittels eines Endotrachealtubus den Fremdkörper in den linken oder rechten Hauptbronchus vorzuschieben [6]. Alternativ kann der Tubus am Fremdkörper vorbeigeschoben werden. Damit wird eine einseitige, endobronchiale Beatmung ermöglicht, und das Kind kann anschließend in die Klinik transportiert und dort unverzüglich der Endoskopie zugeführt werden. 


\section{Fazit für die Praxis}

- Die Fremdkörperverlegung der Atemwege beim Kind stellt eine akut lebensbedrohliche respiratorische Notfallsituation dar. Betroffen sind meist Kleinkinder bis zum Alter von 3 Jahren.

- Esswaren, insbesondere Nüsse, sind die amhäufigsten aspirierten Fremdkörper.

- Typisch für eine Fremdkörperaspiration ist der plötzlich einsetzende Husten und/oder Würgen, ggf. begleitet von Atemnot und Stridor.

- Akut bedrohlich sind im Kehlkopf und in der Trachea gelegene Fremdkörper, welche sehr rasch zu respiratorischer Insuffzienz, Bewusstlosigkeit und Kreislaufstillstand führen können.

- Bei wachen Kindern mit Erstickungsgefahr und ineffektivem Hustenstoß muss mit Rückenschlägen, Thoraxkompression beim Säugling bzw. abdomineller Kompression beim $>1$ Jahr alten Kind versucht werden, den Fremdkörper in eine günstigere Lage zu dislozieren.

- Das bewusstlose, ateminsuffiziente Kind wird beatmet. Wird damit keine Reaktion erreicht, wird mit der Herzmassage bzw. der kardiopulmonalen Reanimation begonnen.

- Der Erfahrene sollte mittels direkter Laryngoskopie einen laryngealen Fremdkörper entfernen bzw. mit einem Endotracheltubus einen trachealen Fremdkörper tiefer in einen Hauptstammbronchus schieben und das Kind unilateral beatmet der Notfallendoskopie zuführen.

\section{Korrespondenzadresse}

Prof. Dr. M. Weiss
Anästhesieabteilung,
Universitäts-Kinderkliniken
Steinwiesstraße 75,

8032 Zürich
Schweiz
markus.weiss@kispi.uzh.ch

Interessenkonflikt. Die korrespondierende Autor gibt an, dass kein Interessenkonflikt besteht.

\section{Literatur}

1. Tomaske M, Gerber AC, Stocker S, Weiss M (2006) Tracheobronchial foreign body aspiration in children diagnostic value of symptoms and signs. Swiss Med Wkly 136:533-538

2. Nicolai T, Reiter K (2004) Notfalltherapie der akuten Fremdkörperaspiration beim Kind. Notfall Rettungsmed 7:501-506

3. Stehen KH, During A, Böwing B (1990) Röntgenzeichen der Fremdkörperaspiration bei Kindern. Radiologe 30:324-327

4. Chavoshzadeh Z, Golnabi A, Rezai N, Mehdizadeh M (2011) Laryngeal foreign body aspiration misdiagnosed as asthma: two case reports an a review of the literature. B-ENT 7:137-140

5. Tomaske M, Gerber AC, Weiss M (2006) Anesthesia and periinterventional morbidity of rigid bronchoscopy for tracheal foreign body diagnosis and removal. Pediatr Anesth 16:123-129

6. Grüber C, Barker M (2009) Akute Atemnot bei Kindern. Notfall Rettungsmed 12:147-156

7. Hoffmann F, Nicolai T(2009) Algorithmus zum Vorgehen bei häufigen respiratorischen Notfällen im Kindesalter. Notfall Rettungsmed 12:576-582

8. Bernhard M, GräsnerJT, Gries A et al (2010) Intraosseous infusion in emergency medicine. Anaesthesiol Intensivmed 51:615-620

9. Biarent D, Bingham R, Eich C et al. (2010) Lebensrettende Massnahmen bei Kindern („Paediatric Life Support $\left.{ }^{\prime \prime}\right)$ Sektion 6 der Leitlinien zur Reanimation 2010 des European Resuscitation Council. Notfall Rettungsmed 13:635-664

\section{Deutscher PHTLS Forschungspreis verliehen}

Anlässlich des Deutschen Interdisziplinären Notfallmedizin Kongresses (DINK) in Wiesbaden hat der Deutsche Berufsverband Rettungsdienst e.V. den 2. PHTLS Forschungspreis für herausragende Tätigkeit auf dem Gebiet der Schwerverletztenforschung verliehen. Der Preis ist mit insgesamt 3500 Euro dotiert und wurde auf drei Arbeiten verteilt. Den 1. Preis gewann die Arbeitsgruppe um Dr. JanThorsten Gräsner aus Kiel. Er hatte anhand des deutschen Reanimationsregisters die Überlebenswahrscheinlichkeit bei Reanimation nach Trauma untersucht. Den 2. Preis erhielt eine Arbeitsgruppe um Dr. Max Skorning aus Aachen. Sie hatten den Einfluss der Telemedizin beim Schwerverletzten anhand eines Simulationsmodells erforscht. Den 3. Preis wurde an Dr. Markus Rössler und seine Kollegen aus Göttingen für eine Untersuchung zur Anwendung der Wirbelsäulenimmobilisation verliehen. Auch im nächsten Jahr wird der Forschungspreis erneut vergeben. Weitere Informationen dazu finden Sie unter www.dbrd.de

Quelle: Deutscher Berufsverband Rettungsdienst e.V. (DBRD), www.dbrd.de 